\title{
K.I.T.T. is real - How conversational AI changes the way we drive (not only for Knight Rider)
}

Patrick Weissert

German Autolabs

This manuscript is not available according to publishing restriction. Thank you for your understanding. 